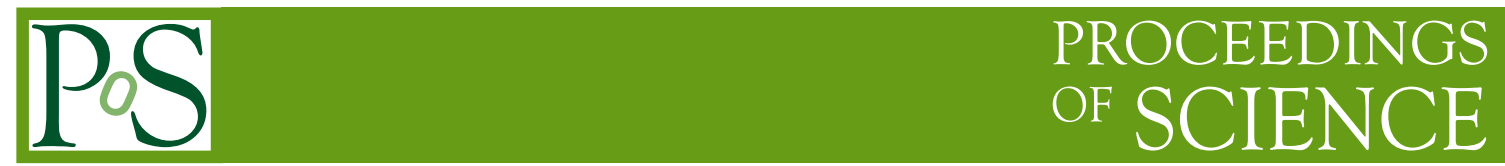

\title{
Higgs measurements at the HL-LHC with CMS
}

\section{Adinda de Wit* on behalf of the CMS collaboration}

Imperial College Sci., Tech. \& Med. (UK)

E-mail: adinda.maite.de.witecern.ch

Prospects for measurements of the properties of the standard model Higgs boson and searches for beyond the standard model Higgs bosons with the CMS experiment at the HL-LHC are presented. The studies are based on projections of existing analyses performed by CMS at $\sqrt{s}=13 \mathrm{TeV}$.

The European Physical Society Conference on High Energy Physics 5-12 July 2017

Venice, Italy

\footnotetext{
${ }^{*}$ Speaker.
} 


\section{Introduction}

In the five years since the discovery of the Higgs boson by the ATLAS and CMS collaborations in $2012[1,2,3]$ the mass of the Higgs boson and its couplings to other particles have been established with remarkable precision using the data collected during Run-1 of the LHC $[4,5]$. Before the start of the high luminosity LHC (HL-LHC) era, the LHC is expected to collect a total integrated luminosity of $300 \mathrm{fb}^{-1}$, with up to $3000 \mathrm{fb}^{-1}$ expected to be collected by the end of the HL-LHC programme. These large datasets will allow for even more precise probing of the Higgs sector.

The CMS detector [6] needs to be upgraded to retain performance at the HL-LHC. At the HL-LHC, the instantaneous luminosity will increase in order for a large dataset to be able to be collected. This leads to an increase in the number of additional interactions per bunch crossing, pileup (PU), to more than 140 on average. Upgrades to the CMS detector planned to function in this environment include increased tracker coverage, increased muon system coverage, the replacement of the calorimeter endcaps with high granularity calorimeters, upgrades to the electronics and scintillators of the barrel calorimeters, and an upgraded trigger system than can cope with higher rates. More information can be found in Ref. [7].

In these proceedings the prospects for standard model (SM) Higgs boson measurements and searches for beyond the standard model (BSM) Higgs bosons with the CMS detector at the HL-LHC will be discussed. The results are based on projections of existing analyses.

\section{Extrapolation strategy}

The results presented in this document are based on extrapolations of analyses using up to $12.9 \mathrm{fb}^{-1}$ of data collected by the CMS experiment during the 2015 and 2016 LHC running periods. Several scenarios for extrapolation are considered [8]. In scenario $\mathbf{S 1}$ the systematic uncertainties are kept constant with integrated luminosity. Scenario $\mathbf{S 1 +}$ is as scenario S1. In addition the effects of high PU and upgrades to the CMS detector on the detector performance are taken into account. In scenario $\mathbf{S 2}$ theoretical uncertainties are halved. Experimental systematic uncertainties are scaled down by the square root of the integrated luminosity, until a lower limit is reached. This lower limit is based on the expected achievable accuracy with the upgraded detector. Scenario $\mathbf{S 2 +}$ is as scenario S2. In addition the effects of high PU and upgrades to the CMS detector on the detector performance are taken into account. A Stat. Only scenario in which only statistical uncertainities are considered is also used.

The modelling of the effects of high PU and upgrades to the CMS detector in the S1+ and S2+ scenarios is analysis-dependent and will be described in the relevant sections below.

\section{Standard model Higgs boson measurements at the HL-LHC}

The projections of standard model Higgs boson measurements presented here comprise the projection of $\mathrm{H} \rightarrow \gamma \gamma$ and $\mathrm{H} \rightarrow \mathrm{ZZ}$ measurements. The original measurements were both based on a dataset corresponding to an integrated luminosity of $12.9 \mathrm{fb}^{-1}$ collected by the CMS experiment 
during the 2016 LHC running period. In addition, projections of searches for SM di-Higgs production are shown. These projections are based on a dataset corresponding to an integrated luminosity of $2.3-2.7 \mathrm{fb}^{-1}$ collected during the 2015 LHC running period.

Figure 1 shows projections of $\mathrm{H} \rightarrow \gamma \gamma$ measurements to 300 and $3000 \mathrm{fb}^{-1}$. For the projection to an integrated luminosity of $3000 \mathrm{fb}^{-1}$ the $\mathrm{S} 1+$ and $\mathrm{S} 2+$ extrapolation scenarios are used. The modifications to model the effects of high PU and upgrades to the CMS detector include a reduction of the vertex identification efficiency and the photon identification efficiency. In figure 1a the projection of the uncertainty on the $\mathrm{H} \rightarrow \gamma \gamma$ signal strength measurement shows that at $3000 \mathrm{fb}^{-1}$ the precision will be limited by the experimental and theoretical uncertainties, which are much larger than the statistical uncertainty. For the projected uncertainty in the fiducial crosssection measurement shown in figure $1 \mathrm{~b}$ the experimental uncertainties also limit the precision at $3000 \mathrm{fb}^{-1}$.

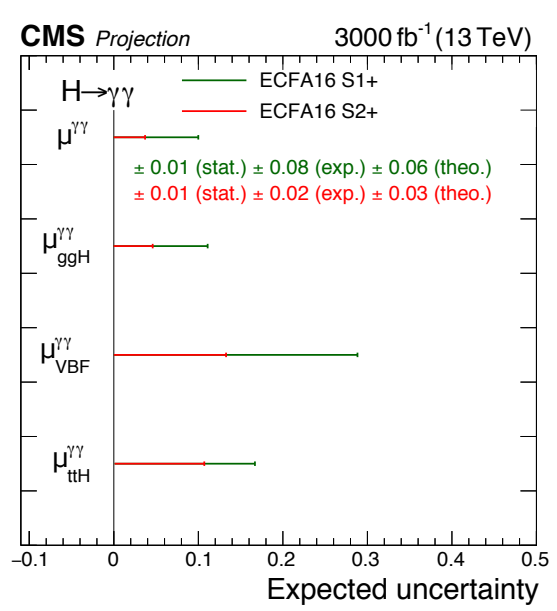

(a)

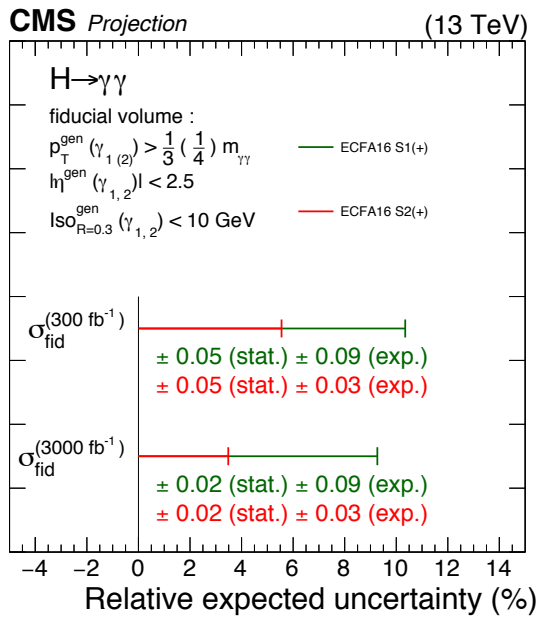

(b)

Figure 1: Projected relative uncertainties on (a) the $\mathrm{H} \rightarrow \gamma \gamma$ signal strength projected to $3000 \mathrm{fb}^{-1}$ and (b) the $\mathrm{H} \rightarrow \gamma \gamma$ fiducial cross section at $300 \mathrm{fb}^{-1}$ and $3000 \mathrm{fb}^{-1}$ [8].

Figure 2 shows projections of $\mathrm{H} \rightarrow \mathrm{ZZ}$ measurements to $3000 \mathrm{fb}^{-1}$ [8]. Extrapolation scenarios $\mathrm{S} 1+$ and $\mathrm{S} 2+$ are used, with the modifications to model the effects of high PU and upgrades to the detector now including effects on the lepton identification efficiency and misidentification rates. Figure 2a shows the projected uncertainties in the signal strength measurement. The uncertainty breakdown again shows that the experimental and theoretical uncertainties dominate over the statistical uncertainties. The projection of the differential fiducial cross-section in figure $2 \mathrm{~b}$ shows a relative uncertainty of 4-10\% varying with scenario and Higgs boson transverse momentum bin.

Projections of searches for non-resonant di-Higgs boson production in several final states were also made. Table 1 shows the expected upper limit on the di-Higgs boson cross-section with respect to the di-Higgs boson production cross-section in the SM at $3000 \mathrm{fb}^{-1}$. 


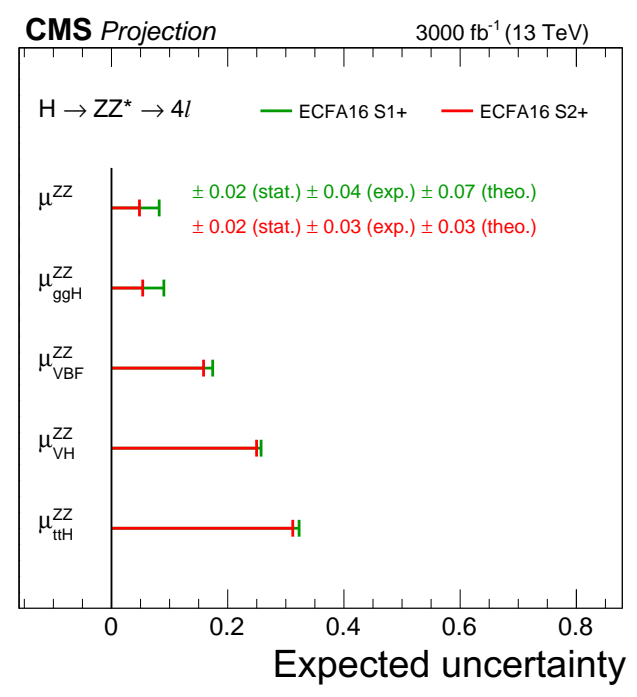

(a)

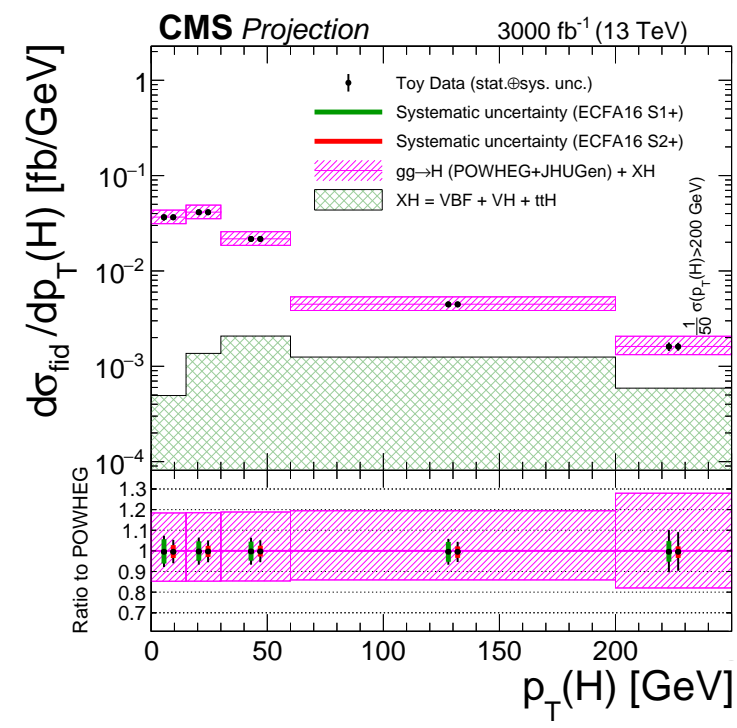

(b)

Figure 2: Projected relative uncertainties on (a) the $\mathrm{H} \rightarrow \mathrm{ZZ}$ signal strength projected to $3000 \mathrm{fb}^{-1}$ and (b) the $\mathrm{H} \rightarrow \mathrm{ZZ}$ differential fiducial cross section at $3000 \mathrm{fb}^{-1}$ [8].

Table 1: Expected upper limits on di-Higgs signal strength at $3000 \mathrm{fb}^{-1}$. Adapted from [8].

\begin{tabular}{llll}
\hline & \multicolumn{3}{c}{ Expected upper limit } \\
Channel & S2 & S2+ & Stat. Only \\
\hline $\mathrm{gg} \rightarrow \mathrm{HH} \rightarrow \gamma \gamma \mathrm{bb}$ & - & 1.44 & 1.37 \\
$\mathrm{gg} \rightarrow \mathrm{HH} \rightarrow \tau \tau \mathrm{bb}$ & 5.2 & - & 3.9 \\
$\mathrm{gg} \rightarrow \mathrm{HH} \rightarrow V V \mathrm{bb}$ & 4.8 & - & 4.6 \\
$\mathrm{gg} \rightarrow \mathrm{HH} \rightarrow \mathrm{bbbb}$ & 7.0 & - & 2.9 \\
\hline
\end{tabular}

\section{Searches for BSM Higgs bosons at the HL-LHC}

Three projections of searches for BSM Higgs bosons are presented here. All of these are based on analyses performed on a dataset corresponding to an integrated luminosity of $2.3 \mathrm{fb}^{-1}$ collected during 2015.

A search for a heavy resonance $\mathrm{X}$ decaying into two $125 \mathrm{GeV}$ Higgs bosons, both decaying to a pair of b-quarks, was projected to an integrated luminosity of $3000 \mathrm{fb}^{-1}$. Such a decay could give access to BSM physics in models with warped extra dimensions [13, 14, 15] or supersymmetry. Table 2 shows the projected expected upper limits on the cross-section times branching ratio of this process. These limits are two orders of magnitude better than those of the reference analysis before projection [9].

A search for a heavy resonance decaying to a pair of tau leptons, performed in the context of the minimal supersymmetric standard model (MSSM) [10, 11], was projected to 300 and $3000 \mathrm{fb}^{-1}$. 
Table 2: Expected upper limits on cross-section times branching ratio of $\mathrm{X} \rightarrow \mathrm{HH} \rightarrow 4 \mathrm{~b}$ at $3000 \mathrm{fb}^{-1}$. Adapted from [8].

\begin{tabular}{lll}
\hline & \multicolumn{2}{c}{ Expected upper limit } \\
$M_{\mathrm{X}}(\mathrm{TeV})$ & $\mathrm{S} 2$ & Stat. Only \\
\hline 0.3 & 46 & 41 \\
0.7 & 7.3 & 3.4 \\
1.0 & 4.4 & 2.4 \\
\hline
\end{tabular}

The projection, interpreted in the $\mathrm{m}_{\mathrm{h}}^{\text {mod+ }}$ MSSM benchmark scenario [12], is shown in figure 3a. The projections are shown alongside the expected results from the reference analysis. The excluded region is significantly larger for the projections. Comparing the different extrapolation scenarios used shows that the projections under S2 and the Stat. Only scenario do not differ in the high mass region. This means the analysis remains statistically limited in this region, even with an integrated luminosity of $3000 \mathrm{fb}^{-1}$.

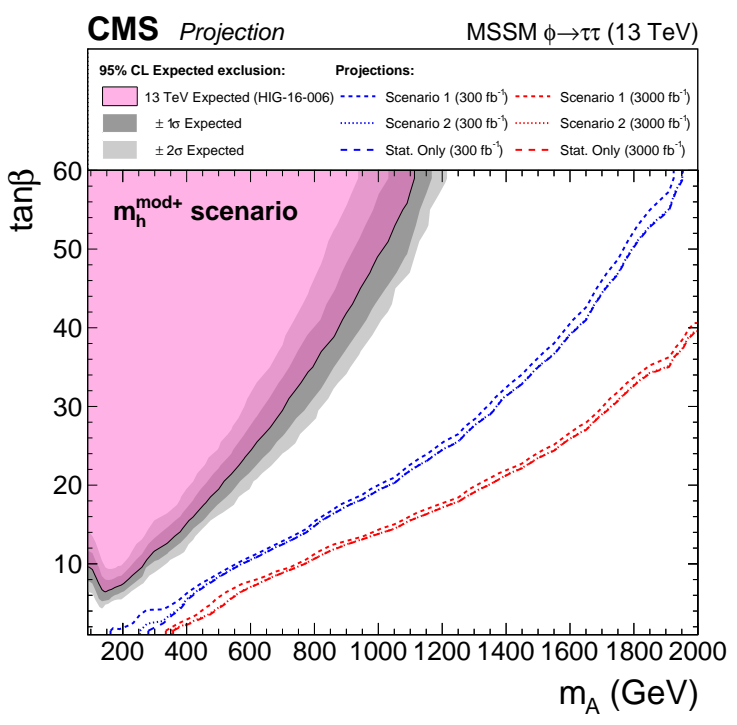

(a)

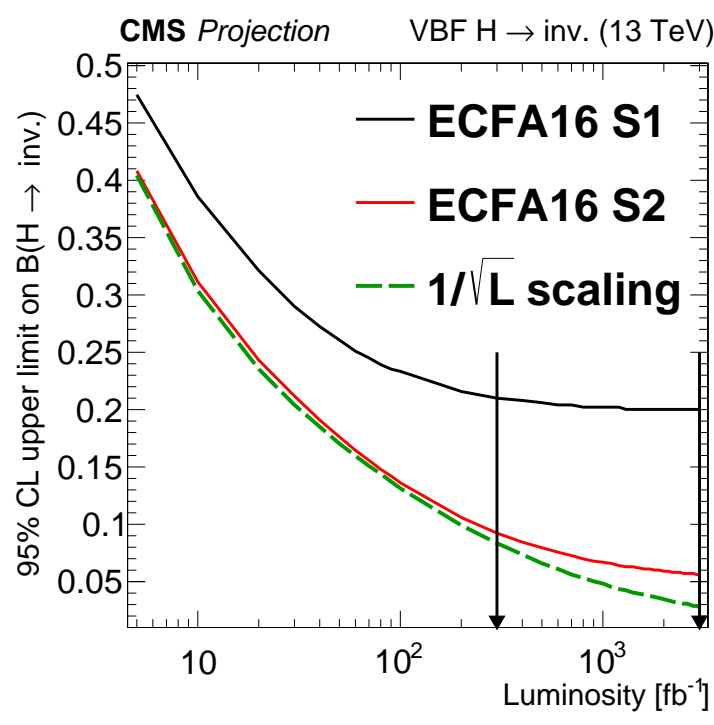

(b)

Figure 3: Projections of searches for BSM Higgs physics. (a) Projection to 300 and $3000 \mathrm{fb}^{-1}$ of the search for a heavy Higgs boson decaying to a pair of tau leptons in the context of the MSSM, interpreted in the $\mathrm{m}_{\mathrm{h}}^{\text {modt }}$ scenario. (b) Projected upper limit on the branching ratio of the $125 \mathrm{GeV}$ Higgs boson decaying invisibly as a function of integrated luminosity. Both figures from Ref. [8].

A search for invisibly decaying $125 \mathrm{GeV}$ Higgs bosons produced via vector boson fusion was projected to various integated luminosities. The results are shown in figure $3 \mathrm{~b}$. In addition to scenarios S1 and S2, an additional extrapolation scenario was employed. The results for this scenario are indicated by the green line. This scenario is similar to $\mathrm{S} 2$, with the exception that no lower bounds are used when scaling the experimental systematic uncertainties down by the square 
root of the integrated luminosity. The results show that with unchanged systematic uncertainties the search becomes systematically limited at $300 \mathrm{fb}^{-1}$. With reduced systematic uncertainties an upper limit on the $\mathrm{H} \rightarrow$ invisible branching ratio of $5 \%$ is achievable at $3000 \mathrm{fb}^{-1}$.

\section{References}

[1] ATLAS Collaboration, "Observation of a new particle in the search for the Standard Model Higgs boson with the ATLAS detector at the LHC", Phys. Lett. B 716 (2012) doi:10.1016/j.physletb.2012.08.020, arXiv:1207. 7214.

[2] CMS Collaboration, "Observation of a new boson at a mass of $125 \mathrm{GeV}$ with the CMS experiment at the LHC", Phys. Lett. B 716 (2012), do i : 10.1016/j.physletb.2012.08.021, arXiv: 1207.7235.

[3] CMS Collaboration, "Observation of a new boson with mass near $125 \mathrm{GeV}$ in pp collisions at sqrt(s) = 7 and 8 TeV", JHEP 06 (2013), do i : 10.1007/JHEP06(2013)081, arXiv: 1303.4571.

[4] ATLAS and CMS Collaborations, "Combined Measurement of the Higgs Boson Mass in pp Collisions at $\sqrt{s}=7$ and $8 \mathrm{TeV}$ with the ATLAS and CMS Experiments", Phys. Rev. Lett. 114 (2015) doi : 10.1103/PhysRevLett.114.191803, arXiv: 1503.07589.

[5] ATLAS and CMS Collaborations, "Measurements of the Higgs boson production and decay rates and constraints on its couplings from a combined ATLAS and CMS analysis of the LHC pp collision data at $\sqrt{s}=7$ and 8 TeV", JHEP 08 (2016), doi:10.1007/JHEP08(2016)045, arXiv: 1606.02266.

[6] CMS Collaboration, "The CMS experiment at the CERN LHC", JINST 3 (2008) do i : 10.1088/1748-0221/3/08/S08004 .

[7] CMS Collaboration, "Technical Proposal for the Phase-II Upgrade of the CMS Detector", Technical Report CERN-LHCC-2015-010. LHCC-P-008. CMS-TDR-15-02 (2015). https://cds.cern.ch/record/2020886.

[8] CMS Collaboration, "Projected performance of Higgs analyses at the HL-LHC for ECFA 2016", CMS-PAS-FTR-16-002, CERN (2016). https://cds.cern. ch/record/2266165.

[9] CMS Collaboration, "Search for resonant pair production of Higgs bosons decaying to two bottom quark-antiquark pairs in proton-proton collisons at $13 \mathrm{TeV}$ ",

CMS-PAS-HIG-16-002, CERN (2016). https : / / cds . cern. ch/record/2141024.

[10] P. Fayet, "Supergauge invariant extension of the Higgs mechanism and a model for the electron and its neutrino", Nucl. Phys. B90 (1975) 104, doi : 10.1016/0550-3213(75) 90636-7.

[11] P. Fayet, "Spontaneously broken supersymmetric theories of weak, electromagnetic and strong interactions", Phys. Lett. B69 (1977) 489, do i : 10.1016/0370-2693(77)90852-8.

[12] P. Bechtle et al., "Probing the Standard Model with Higgs signal rates from the Tevatron, the LHC and a future ILC", JHEP 11 (2014) 039, do : : 10.1007/JHEP11(2014)039, arXiv: 1403.1582 .

[13] W.D. Goldberger and M.S. Wise, "Modulus stabilization with bulk fields", Phys.Rev.Lett. 83 (1999) 33703373. 
[14] C. Csaki, J. Hubisz, and S.J. Lee, "Radion phenomenology in realistic warped space Model", Phys. Rev. D 62 (2000) 045015.

[15] H.Davoudiasl, J.Hewett, and T. Rizzo, "Phenomenology of the Randall-Sundrum Gauge Hierarchy Model", Phys. Rev. Lett. 84 (2000) 2080. 